\title{
Assessing Apps for Patients with Genitourinary Tumors Using the Mobile Application Rating Scale (MARS): Systematic Search in App Stores and Content Analysis
}

Miguel Ángel Amor-García, PharmD; Roberto Collado-Borrell, PhD, PharmD; Vicente Escudero-Vilaplana, PhD, PharmD; Alejandra Melgarejo-Ortuño, PharmD; Ana Herranz-Alonso, PharmD, PhD; José Ángel Arranz Arija, MD, PhD; María Sanjurjo-Sáez, PharmD

Hospital General Universitario Gregorio Marañón, Instituto de Investigación Sanitaria Gregorio Marañón, Madrid, Spain

\section{Corresponding Author:}

Vicente Escudero-Vilaplana, PhD, PharmD

Hospital General Universitario Gregorio Marañón

Instituto de Investigación Sanitaria Gregorio Marañón

Doctor Esquerdo, 46

Madrid, 28007

Spain

Phone: 34915867714

Fax: 34915866621

Email: vicente.escudero@salud.madrid.org

\section{Abstract}

Background: The large number of available cancer apps and their impact on the population necessitates a transparent, objective, and comprehensive evaluation by app experts, health care professionals, and users. To date, there have been no analyses or classifications of apps for patients with genitourinary cancers, which are among the most prevalent types of cancer.

Objective: The objective of our study was to analyze the quality of apps for patients diagnosed with genitourinary cancers using the Mobile Application Rating Scale (MARS) and identify high-quality apps.

Methods: We performed an observational cross-sectional descriptive study of all smartphone apps for patients diagnosed with genitourinary cancers available on iOS and Android platforms. In July 2019, we searched for all available apps for patients with genitourinary cancers (bladder, prostate, cervical, uterine, endometrial, kidney, testicular, and vulvar) or their caregivers. Apps were downloaded and evaluated, and the general characteristics were entered into a database. The evaluation was performed by 2 independent researchers using the MARS questionnaire, which rates 23 evaluation criteria clustered in 5 domains (Engagement, Functionality, Esthetics, Information, and Subjective Quality) on a scale from 1 to 5.

Results: In total, 46 apps were analyzed. Of these, 31 (67\%) were available on Android, 6 (13\%) on iOS, and 9 (20\%) on both platforms. The apps were free in $89 \%$ of cases $(41 / 46)$, and $61 \%(28 / 46)$ had been updated in the previous year. The apps were intended for prostate cancer in $30 \%$ of cases (14/46) and cervical cancer in 17\% (8/46). The apps were mainly informative (63\%, 29/46), preventive $(24 \%, 11 / 46)$, and diagnostic (13\%, 6/46). Only $7 / 46$ apps (15\%) were developed by health care organizations. The mean MARS score for the overall quality of the 46 apps was 2.98 (SD 0.77), with a maximum of 4.63 and a minimum of 1.95. Functionality scores were quite similar for most of the apps, with the greatest differences in Engagement and Esthetics, which showed acceptable scores in one-third of the apps. The 5 apps with the highest MARS score were the following: "Bladder cancer manager," "Kidney cancer manager," "My prostate cancer manager," "Target Ovarian Cancer Symptoms Diary," and "My Cancer Coach." We observed statistically significant differences in the MARS score between the operating systems and the developer types $(P<.001$ and $P=.01$, respectively), but not for cost $(P=.62)$.

Conclusions: MARS is a helpful methodology to decide which apps can be prescribed to patients and to identify which features should be addressed to improve these tools. Most of the apps designed for patients with genitourinary cancers only try to provide data about the disease, without coherent interactivity. The participation of health professionals in the development of these apps is low; nevertheless, we observed that both the participation of health professionals and regular updates were correlated with quality.

(JMIR Mhealth Uhealth 2020;8(7):e17609) doi: $\underline{10.2196 / 17609}$ 


\section{KEYWORDS}

genitourinary cancer; mobile apps; eHealth; mHealth; rating tool

\section{Introduction}

Genitourinary cancers represent $25 \%$ of all types of cancer [1]. For men, the most commonly diagnosed cancers are prostate cancer and bladder cancer, while for women, cervical cancer and uterine cancer are the most common [2]. Although these cancers are leading causes of death, recent advances in diagnosis and therapy have led to significant improvements in the overall survival of these patients [1].

The chronification of many genitourinary cancers and the special features of newer treatments, such as oral anticancer agents and immunotherapy, have also changed the profile of patients with this type of cancer [3-5]. As a result of the more exhaustive amount of information that patients and relatives need to know about the characteristics of the disease and treatment, there are new challenges for improving communication between patients and caregivers, and greater opportunities for contact with health care professionals. In this regard, information and communications technologies, especially mobile apps and remote assistance services [6-8], could help to improve the autonomy and communication options of these patients [9].

Currently, more than 200 health apps are released daily, and in the last 2 years, the number of available apps has doubled to reach more than 300,000 [10]. This development, which has not been specifically regulated, has led to the diffusion of some poor-quality apps $[11,12]$. Apps are downloaded from one of the operating system stores ("Play Store" for Android and "App Store" for iOS), where they are valued based on only 2 criteria (ie, the number of downloads and the user ratings) [13,14]. They are nonspecific search engines that do not enable the user to apply filters to assess their disease, the purpose of the app, or the quality of the app [6]. Consequently, searching for high-quality information is becoming even more difficult, with the result that the user downloads apps of uncertain reliability that are likely not the most appropriate option for his/her needs [15-17]. This aspect is particularly important for patients with cancer, where receiving poor-quality information may have a negative impact on prognosis [18].

The large number of available health care apps and their impact on the population necessitates a transparent, objective, and comprehensive evaluation by app experts, health care professionals, and users $[15,19,20]$. There are several methods to evaluate the quality of health apps. The most appropriate for use in online stores where patients can search and contrast health care apps is the Mobile Application Rating Scale (MARS). This tool provides a simple, quantitative, and validated system that enables rapid evaluation with little variation [12,13,21]. The number of apps for patients with cancer is continuously increasing owing to the availability of new information, requirements for communication, and the empowerment of patients who wish to participate in their care [5,9]. However, there is no standardized methodology for the classification, assessment, and validation of apps for patients with

genitourinary cancers, although MARS is the most widely recommended.

The objective of our study was to analyze the quality of apps for patients diagnosed with genitourinary cancers using the MARS scale to identify high-quality apps.

\section{Methods}

\section{Study Design}

We performed an observational cross-sectional descriptive study of all smartphone apps for patients diagnosed with genitourinary cancers available on the $\mathrm{iOS}$ and Android platforms.

Our study followed a methodology to select the apps and adhered to the PRISMA (Preferred Reporting Items for Systematic review and Meta-Analysis Protocols) guidelines [22]. In July 2019, a search was conducted in the App Store (iOS) and Play Store (Android) within the categories "medicine" and "health and fitness." The terms used in this search were the following: "bladder cancer," "kidney cancer," "testicular cancer," and "prostate cancer" for urological tumors and "reproductive cancer," "endometrial cancer," "cervical cancer," "uterine cancer," "ovarian cancer," and "vulvar cancer" for gynecological tumors.

Once the search was completed, all available information on the platform was analyzed, and only apps that were in English or Spanish and intended for patients and caregivers were selected. For this study, we excluded apps aimed specifically at health care professionals, those for charitable purposes or without scientific content, and those not specific to genitourinary cancers. Apps that met the indicated criteria were downloaded and evaluated, regardless of cost. The iOS apps were downloaded to an iPhone 8 (version 12.3.2) and the Android apps were downloaded to a Xiaomi Mi A1 (version 9.0).

\section{Characteristics and Content of the Apps}

The general characteristics of the applications were entered into a database. Recorded characteristics included the name, platform (Android or iOS), cost $(€)$, category (medicine and health and fitness), date of the last update, language, and target type of cancer. The content of the applications was classified into 1 of 3 categories according to its purpose: informative, preventive, and diagnostic. Furthermore, any information about the participation of health professionals in the app design or development was included. Qualified professionals were considered to have contributed to the app contents if the app had been developed by health care organizations such as local health authorities, universities, scientific societies and foundations, and hospitals.

\section{MARS Evaluation}

The quality of the apps was then assessed using MARS. This methodology includes 23 evaluation criteria, clustered within 5 domains: (1) "Engagement," which assesses the entertainment, customization, and interactivity of the app (feedback, reminders, and notifications); (2) "Functionality," which examines the 
functionality of the app, ease of use, transition between screens, and intuitive design; (3) "Esthetics," which assesses graphic design, visual appeal, and stylistic consistency; (4) "Information," which evaluates the quality of the content (text, measures, and references), determined by the credibility of the source; and (5) "Subjective quality," which determines whether the app could be recommended to people who might benefit from it, if they would be prepared to pay for it, how many times it would be used, and what overall star rating it would be given. Each evaluation criterion was rated from 1 to 5 (1=Inadequate, 2=Poor, 3=Acceptable, 4=Good, 5=Excellent) [12,13,22]. The MARS evaluation was carried out by 2 independent researchers with experience in app design and development and familiarity with genitourinary cancers. After all the evaluation criteria were scored, the mean score of the domains was calculated to obtain the total mean MARS score, which describes the overall quality of the app.

Figure 1. Study flowchart. MARS: Mobile Application Rating Scale.
The quantitative variables were described using mean and standard deviation. The categorical variables were described using frequencies and percentages. The numerical variables were compared using the $t$ test. A weighted Cohen $\kappa$ test was performed to guarantee the reliability of those data analyzed by 2 independent observers, and the joint probability agreement (estimated as the percentage of times the raters agreed on an item) was measured. Results with a $P$ value $<.05$ were considered statistically significant. Data were analyzed using Stata (version IC-15, StataCorp LLC).

\section{Results}

\section{Study Design}

The app search provided a total of 1055 apps, of which 51 were finally downloaded (Figure 1).
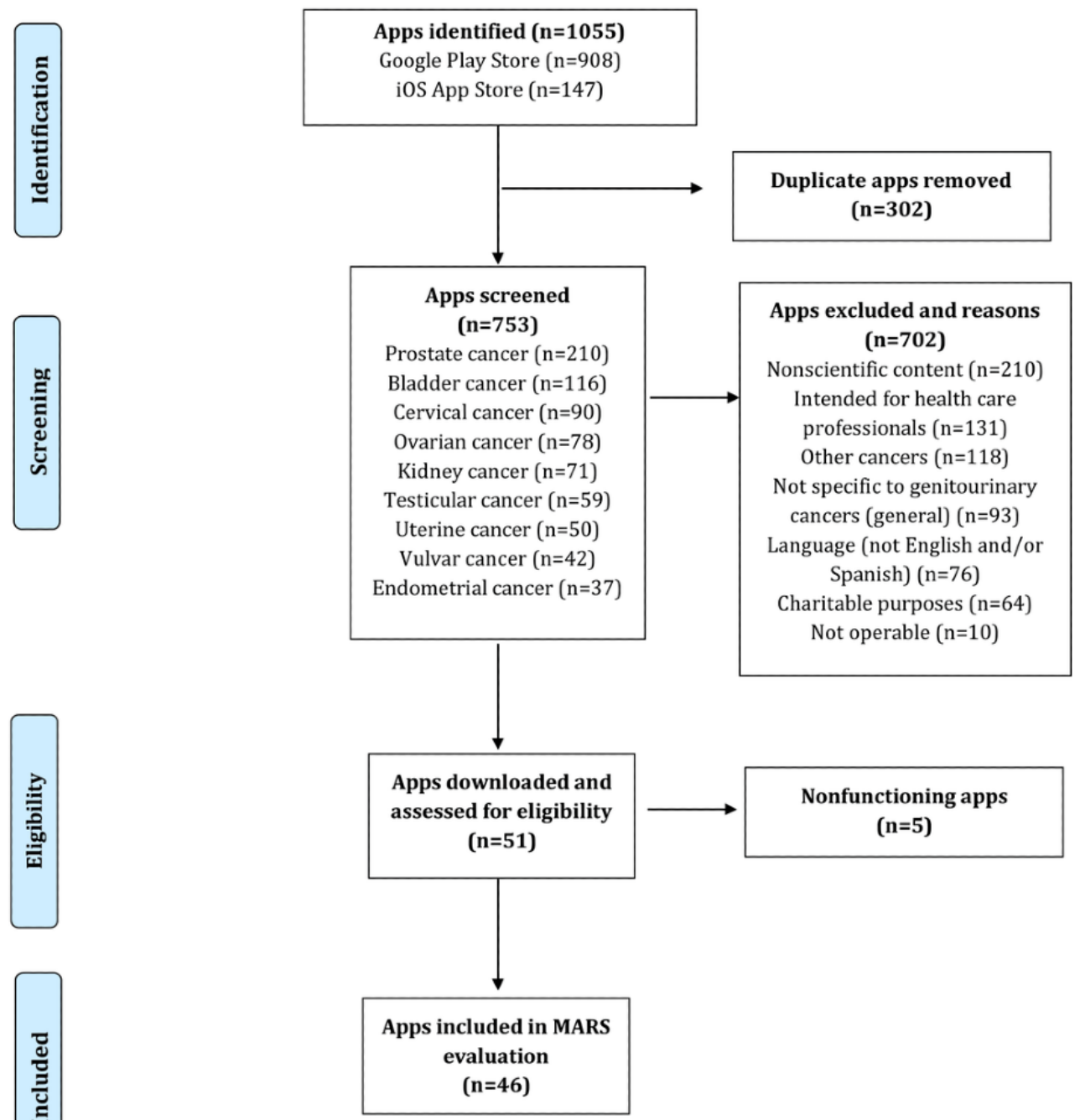

\section{Characteristics and Content of the Apps}

At the time of the MARS evaluation, 5 of those 51 applications had been removed from the store or could not be opened, leading to a final total of 46 . Of these, $31(67 \%)$ were available on Android, $6(13 \%)$ on iOS, and $9(20 \%)$ on both platforms. Most of the apps $(89 \%, 41 / 46)$ were free, with only 5 apps $(11 \%$, $5 / 46$ ) requiring payment (mean cost of $€ 3.51$ [US \$3.98], SD 
1.21). The general characteristics of the apps are shown in Table 1. Table 2 describes the characteristics of the apps.

Most of the apps were informative $(63 \%, 29 / 46)$, followed by preventive $(24 \%, 11 / 46)$ and diagnostic $(13 \%, 6 / 46)$. Only 7 of the 46 apps (15\%) were developed by health care organizations, which included 3 scientific societies (43\%), 3 universities (43\%), and 1 hospital (14\%). Regarding the type of cancer, 27/46 (59\%) were intended for patients with urological cancers and 21/46
(47\%) for those with gynecologic cancers. Apps for urological cancers were intended for prostate cancer in 14/46 apps (30\%), testicular cancer in $7 / 46(15 \%)$, bladder cancer in $4 / 46(9 \%)$, and kidney cancer in 2/46 (4\%). Gynecologic cancers were represented by cervical cancer in 9/46 apps (20\%), ovarian cancer in $8 / 46(15 \%)$, uterine cancer in $2 / 46(4 \%)$, endometrial cancer in $2 / 46(4 \%)$, and vulvar cancer in $1 / 46(2 \%)$. One app contained information on cervical, testicular, and ovarian cancer.

Table 1. General characteristics of the apps.

\begin{tabular}{|c|c|}
\hline Characteristics & Apps, n (\%) \\
\hline \multicolumn{2}{|l|}{ Platform } \\
\hline Android & $31(67)$ \\
\hline Android/iOS & $9(20)$ \\
\hline iOS & $6(13)$ \\
\hline \multicolumn{2}{|l|}{ Cost } \\
\hline No & $41(89)$ \\
\hline Yes & $5(11)$ \\
\hline \multicolumn{2}{|l|}{ Category } \\
\hline Medicine & $26(57)$ \\
\hline Health and fitness & $20(43)$ \\
\hline \multicolumn{2}{|l|}{ Year of the last update } \\
\hline 2014 & $1(2)$ \\
\hline 2015 & $3(7)$ \\
\hline 2016 & $2(4)$ \\
\hline 2017 & $12(26)$ \\
\hline 2018 & $28(61)$ \\
\hline \multicolumn{2}{|l|}{ Language } \\
\hline English & $43(94)$ \\
\hline Spanish & $2(4)$ \\
\hline English/Spanish & $1(2)$ \\
\hline
\end{tabular}


Table 2. Characteristics of the apps analyzed ${ }^{\mathrm{a}}$.

\begin{tabular}{|c|c|c|c|c|c|c|c|c|c|c|c|}
\hline \multirow[t]{2}{*}{ Name of the app } & \multirow[t]{2}{*}{ Type of cancer } & \multicolumn{3}{|c|}{ Purpose $^{b}$} & \multicolumn{2}{|c|}{ Platform } & \multirow[t]{2}{*}{ Free } & \multirow{2}{*}{$\begin{array}{l}\text { Updated in } \\
\text { the last year }\end{array}$} & \multirow{2}{*}{$\begin{array}{l}\text { Developed by a } \\
\text { health organization }\end{array}$} & \multicolumn{2}{|c|}{ Language $^{c}$} \\
\hline & & I & $\mathrm{P}$ & $\mathrm{D}$ & iOS & Android & & & & $\mathrm{E}$ & $S$ \\
\hline Ball Checker & Testicular & & & $\checkmark$ & $\checkmark$ & $\checkmark$ & $\checkmark$ & $\checkmark$ & $\checkmark$ & & $\checkmark$ \\
\hline $\begin{array}{l}\text { Best Prostate Cancer } \\
\text { Treatment }\end{array}$ & Prostate & $\checkmark$ & & & $\checkmark$ & & $\checkmark$ & $\checkmark$ & & $\checkmark$ & \\
\hline $\begin{array}{l}\text { Bladder cancer (Bedie- } \\
\text { man) }\end{array}$ & Bladder & $\checkmark$ & & & & $\checkmark$ & $\checkmark$ & $\checkmark$ & & $\checkmark$ & \\
\hline Bladder cancer manager & Bladder & & $\checkmark$ & & $\checkmark$ & & $\checkmark$ & $\checkmark$ & & $\checkmark$ & \\
\hline Cancer cervix fact & Cervical & $\checkmark$ & & & & $\checkmark$ & $\checkmark$ & $\checkmark$ & & $\checkmark$ & \\
\hline $\begin{array}{l}\text { Cáncer de cuello uteri- } \\
\text { no }\end{array}$ & Cervical & $\checkmark$ & & & & $\checkmark$ & $\checkmark$ & & & $\checkmark$ & \\
\hline Cáncer de ovarios & Ovarian & $\checkmark$ & & & & $\checkmark$ & $\checkmark$ & & & $\checkmark$ & \\
\hline $\begin{array}{l}\text { Cancer de prostata } \\
\text { (Anastore) }\end{array}$ & Prostate & $\checkmark$ & & & & $\checkmark$ & $\checkmark$ & & & $\checkmark$ & \\
\hline $\begin{array}{l}\text { Cancer de prostata (Pen } \\
\text { Drouzi) }\end{array}$ & Prostate & $\checkmark$ & & & & $\checkmark$ & $\checkmark$ & $\checkmark$ & & $\checkmark$ & \\
\hline Cancer de RIÑON & Kidney & $\checkmark$ & & & & $\checkmark$ & $\checkmark$ & & & $\checkmark$ & \\
\hline Cáncer de vejiga & Bladder & $\checkmark$ & & & & $\checkmark$ & $\checkmark$ & & & $\checkmark$ & \\
\hline $\begin{array}{l}\text { Cáncer testicular } \\
\text { (Anass apps) }\end{array}$ & Testicular & $\checkmark$ & & & & $\checkmark$ & $\checkmark$ & & & $\checkmark$ & \\
\hline $\begin{array}{l}\text { Cancer testicular (Ex- } \\
\text { pert Health Studio) }\end{array}$ & Testicular & $\checkmark$ & & & & $\checkmark$ & $\checkmark$ & $\checkmark$ & & $\checkmark$ & \\
\hline $\begin{array}{l}\text { Cancer testicular } \\
\text { (Health Advice Ideas) }\end{array}$ & Testicular & $\checkmark$ & & & & $\checkmark$ & $\checkmark$ & $\checkmark$ & & $\checkmark$ & \\
\hline $\begin{array}{l}\text { Cancer testicular (Pen } \\
\text { Drouzi) }\end{array}$ & Testicular & $\checkmark$ & & & & $\checkmark$ & $\checkmark$ & $\checkmark$ & & $\checkmark$ & \\
\hline Cáncer uterino & Uterine & $\checkmark$ & & & & $\checkmark$ & $\checkmark$ & & & $\checkmark$ & \\
\hline $\begin{array}{l}\text { Cervical cancer (Bedie- } \\
\text { man) }\end{array}$ & Cervical & $\checkmark$ & & & & $\checkmark$ & $\checkmark$ & $\checkmark$ & & $\checkmark$ & \\
\hline $\begin{array}{l}\text { Cervical cancer (Natu- } \\
\text { ral health care) }\end{array}$ & Cervical & $\checkmark$ & & & & $\checkmark$ & $\checkmark$ & $\checkmark$ & & $\checkmark$ & \\
\hline $\begin{array}{l}\text { Cervical cancer } \\
\text { (Nougat spring) }\end{array}$ & Cervical & $\checkmark$ & & & & $\checkmark$ & $\checkmark$ & $\checkmark$ & & $\checkmark$ & \\
\hline $\begin{array}{l}\text { Cervical cancer (Person- } \\
\text { al Remedies LLC) }\end{array}$ & Cervical & & $\checkmark$ & & $\checkmark$ & $\checkmark$ & & $\checkmark$ & & $\checkmark$ & \\
\hline $\begin{array}{l}\text { Common causes of cer- } \\
\text { vical cancer }\end{array}$ & Cervical & & $\checkmark$ & & & $\checkmark$ & $\checkmark$ & & & $\checkmark$ & \\
\hline El cancer de vulva & Vulvar & $\checkmark$ & & & & $\checkmark$ & $\checkmark$ & & & $\checkmark$ & \\
\hline $\begin{array}{l}\text { Endometrial cancer } \\
\text { (Bedieman) }\end{array}$ & Endometrial & $\checkmark$ & & & & $\checkmark$ & $\checkmark$ & $\checkmark$ & & $\checkmark$ & \\
\hline $\begin{array}{l}\text { Endometrial cancer } \\
\text { (online Global Groups) }\end{array}$ & Endometrial & $\checkmark$ & & & & $\checkmark$ & $\checkmark$ & $\checkmark$ & & $\checkmark$ & \\
\hline Global Pap App & Cervical & & $\checkmark$ & & $\checkmark$ & & $\checkmark$ & $\checkmark$ & & $\checkmark$ & \\
\hline $\begin{array}{l}\text { How to prevent ovarian } \\
\text { cancer }\end{array}$ & Ovarian & $\checkmark$ & & & & $\checkmark$ & $\checkmark$ & $\checkmark$ & & $\checkmark$ & \\
\hline $\begin{array}{l}\text { IPCRC (Prostate Ca } \\
\text { Calculator) }\end{array}$ & Prostate & & & $\checkmark$ & & $\checkmark$ & $\checkmark$ & & & $\checkmark$ & \\
\hline $\begin{array}{l}\text { itsaMANTHING- } \\
\text { Prostate cancer }\end{array}$ & Prostate & $\checkmark$ & & & $\checkmark$ & $\checkmark$ & $\checkmark$ & & $\checkmark$ & $\checkmark$ & \\
\hline
\end{tabular}




\begin{tabular}{|c|c|c|c|c|c|c|c|c|c|c|c|}
\hline \multirow[t]{2}{*}{ Name of the app } & \multirow[t]{2}{*}{ Type of cancer } & \multicolumn{3}{|c|}{ Purpose $^{b}$} & \multicolumn{2}{|c|}{ Platform } & \multirow[t]{2}{*}{ Free } & \multirow{2}{*}{$\begin{array}{l}\text { Updated in } \\
\text { the last year }\end{array}$} & \multirow{2}{*}{$\begin{array}{l}\text { Developed by a } \\
\text { health organization }\end{array}$} & \multicolumn{2}{|c|}{ Language $^{c}$} \\
\hline & & I & $P$ & $\mathrm{D}$ & iOS & Android & & & & E & $\mathrm{s}$ \\
\hline Kidney cancer manager & Kidney & & $\checkmark$ & & $\checkmark$ & & $\checkmark$ & $\checkmark$ & & $\checkmark$ & \\
\hline My Cancer Coach & Prostate & $\checkmark$ & & & & $\checkmark$ & $\checkmark$ & $\checkmark$ & $\checkmark$ & $\checkmark$ & \\
\hline $\begin{array}{l}\text { My prostate cancer } \\
\text { manager }\end{array}$ & Prostate & & $\checkmark$ & & $\checkmark$ & & $\checkmark$ & $\checkmark$ & & $\checkmark$ & \\
\hline $\begin{array}{l}\text { OddBalls-Check Your- } \\
\text { self }\end{array}$ & Testicular & & $\checkmark$ & & & $\checkmark$ & $\checkmark$ & & & $\checkmark$ & \\
\hline $\begin{array}{l}\text { Ovarian cancer (Person- } \\
\text { al Remedies LLC) }\end{array}$ & Ovarian & & $\checkmark$ & & $\checkmark$ & $\checkmark$ & & $\checkmark$ & & $\checkmark$ & \\
\hline $\begin{array}{l}\text { Ovarian Cancer Aware- } \\
\text { ness }\end{array}$ & Ovarian & $\checkmark$ & & & & $\checkmark$ & $\checkmark$ & & & $\checkmark$ & \\
\hline $\begin{array}{l}\text { Ovarian cancer symp- } \\
\text { toms diary }\end{array}$ & Ovarian & & $\checkmark$ & & $\checkmark$ & $\checkmark$ & $\checkmark$ & & $\checkmark$ & $\checkmark$ & \\
\hline $\begin{array}{l}\text { Prostate cancer (Dina- } \\
\text { tale) }\end{array}$ & Prostate & $\checkmark$ & & & & $\checkmark$ & $\checkmark$ & $\checkmark$ & & $\checkmark$ & \\
\hline $\begin{array}{l}\text { Prostate cancer (Person- } \\
\text { al Remedies LLC) }\end{array}$ & Prostate & & $\checkmark$ & & $\checkmark$ & $\checkmark$ & & $\checkmark$ & & $\checkmark$ & \\
\hline $\begin{array}{l}\text { Prostate Cancer Calcula- } \\
\text { tor }\end{array}$ & Prostate & & & $\checkmark$ & & $\checkmark$ & $\checkmark$ & & & $\checkmark$ & \\
\hline Prostate PRO-Tracker & Prostate & & & $\checkmark$ & $\checkmark$ & & $\checkmark$ & & $\checkmark$ & $\checkmark$ & \\
\hline ProstateCheck & Prostate & & & $\checkmark$ & $\checkmark$ & $\checkmark$ & & & $\checkmark$ & & $\checkmark$ \\
\hline Reproductive cancers & $\begin{array}{l}\text { Ovarian, cervical, } \\
\text { testicular }\end{array}$ & $\checkmark$ & & & & $\checkmark$ & $\checkmark$ & $\checkmark$ & & $\checkmark$ & \\
\hline $\begin{array}{l}\text { Rotterdam Prostate } \\
\text { Cancer Risk }\end{array}$ & Prostate & & & $\checkmark$ & $\checkmark$ & $\checkmark$ & & $\checkmark$ & $\checkmark$ & $\checkmark$ & $\checkmark$ \\
\hline $\begin{array}{l}\text { Target Ovarian Cancer } \\
\text { Symptoms Diary }\end{array}$ & Ovarian & & $\checkmark$ & & $\checkmark$ & $\checkmark$ & $\checkmark$ & $\checkmark$ & & $\checkmark$ & \\
\hline Treat prostate cancer & Prostate & $\checkmark$ & & & & $\checkmark$ & $\checkmark$ & & & $\checkmark$ & \\
\hline Treating bladder cancer & Bladder & $\checkmark$ & & & & $\checkmark$ & $\checkmark$ & $\checkmark$ & & $\checkmark$ & \\
\hline $\begin{array}{l}\text { Ways to treat uterine } \\
\text { cancer }\end{array}$ & Uterine & $\checkmark$ & & & & $\checkmark$ & $\checkmark$ & $\checkmark$ & & $\checkmark$ & \\
\hline
\end{tabular}

${ }^{\mathrm{a}}$ In apps with the same name, the developer is indicated in parentheses.

${ }^{\mathrm{b}} \mathrm{I}$ : informative; P: preventive; D: diagnostic.

${ }^{\mathrm{c}}$ E: English; S: Spanish.

\section{MARS Evaluation}

The mean MARS score for the overall quality of the 46 apps was 2.98 (SD 0.77), with a maximum of 4.63 and a minimum of 1.95 (Table 3 ).

The Functionality scores were similar for most of the apps. The apps provided adequate and rapid movement between the screens and menus. The greatest differences were found in the Engagement domain because of deficiencies in areas such as customization and interactivity. Similar differences were found in the Information domain because of the visual explanation and evidence base. The Esthetics domain showed acceptable scores in one-third of the apps; this was associated with a greater update rate and visual appeal. 
Table 3. Mobile Application Rating Scale scores of the evaluated apps out of $5^{\mathrm{a}}$.

\begin{tabular}{|c|c|c|c|c|c|c|}
\hline Name of app & Engagement & Functionality & Esthetics & Information & $\begin{array}{l}\text { Subjective } \\
\text { quality }\end{array}$ & Overall \\
\hline Bladder cancer manager & 4.60 & 4.63 & 4.83 & 4.57 & 4.50 & 4.63 \\
\hline Kidney cancer manager & 4.60 & 4.63 & 4.83 & 4.57 & 4.50 & 4.63 \\
\hline My prostate cancer manager & 4.60 & 4.63 & 4.83 & 4.57 & 4.50 & 4.63 \\
\hline Target Ovarian Cancer Symptoms Diary & 4.10 & 4.75 & 4.83 & 3.93 & 3.63 & 4.25 \\
\hline My Cancer Coach & 4.10 & 4.63 & 3.67 & 4.50 & 4.25 & 4.23 \\
\hline Rotterdam Prostate Cancer Risk & 3.20 & 4.63 & 3.67 & 4.43 & 3.25 & 3.83 \\
\hline ProstateCheck & 3.40 & 4.88 & 3.50 & 3.36 & 3.25 & 3.68 \\
\hline Ball Checker & 3.50 & 4.25 & 3.67 & 4.21 & 2.75 & 3.68 \\
\hline Prostate PRO-Tracker & 3.40 & 4.75 & 3.67 & 4.21 & 2.88 & 3.64 \\
\hline Global Pap App & 2.90 & 4.88 & 3.67 & 3.71 & 3.00 & 3.63 \\
\hline OddBalls - Check yourself & 3.30 & 3.88 & 3.50 & 4.00 & 3.25 & 3.59 \\
\hline Prostate Cancer Calculator & 3.80 & 4.63 & 2.00 & 3.71 & 3.5 & 3.53 \\
\hline itsaMANTHING-Prostate Cancer & 2.60 & 4.88 & 3.17 & 3.71 & 3.13 & 3.50 \\
\hline $\begin{array}{l}\text { Endometrial cancer (online Global } \\
\text { Groups) }\end{array}$ & 2.60 & 4.63 & 3.50 & 3.29 & 3.25 & 3.45 \\
\hline Prostate cancer (Dinatale) & 2.50 & 4.63 & 3.67 & 3.64 & 2.38 & 3.36 \\
\hline IPCRC (Prostate Ca Calculator) & 2.50 & 4.88 & 3.50 & 2.71 & 3.00 & 3.32 \\
\hline Treating bladder cancer & 2.50 & 4.50 & 3.67 & 3.29 & 2.63 & 3.32 \\
\hline Ovarian Cancer Symptoms Diary & 2.90 & 4.38 & 3.17 & 3.14 & 2.88 & 3.29 \\
\hline Cáncer de prostata (Pen Drouzi) & 1.90 & 4.50 & 3.50 & 3.36 & 2.25 & 3.10 \\
\hline Cáncer testicular (Pen Drouzi) & 1.90 & 4.50 & 3.50 & 3.36 & 3.25 & 3.10 \\
\hline Cervical cancer (Nougat spring) & 2.20 & 4.75 & 2.50 & 2.93 & 2.75 & 3.03 \\
\hline Best Prostate Cancer Treatment & 2.40 & 4.00 & 3.33 & 3.21 & 2.13 & 3.02 \\
\hline Bladder cancer & 2.10 & 4.63 & 3.00 & 2.86 & 2.38 & 2.99 \\
\hline Cervical cancer (Personal Remedies LLC) & 3.20 & 2.25 & 3.50 & 2.86 & 1.88 & 2.74 \\
\hline Ovarian cancer (Personal Remedies LLC) & 3.20 & 2.25 & 3.50 & 2.86 & 1.88 & 2.74 \\
\hline Prostate cancer (Personal Remedies LLC) & 3.20 & 2.25 & 3.50 & 2.86 & 1.88 & 2.74 \\
\hline Cancer testicular (Expert Health Studio) & 1.80 & 3.50 & 3.33 & 3.14 & 1.63 & 2.68 \\
\hline Cancer testicular (Health Advice Ideas) & 1.80 & 3.38 & 3.33 & 3.14 & 1.50 & 2.63 \\
\hline Reproductive cancers & 1.60 & 4.50 & 3.00 & 2.43 & 1.50 & 2.61 \\
\hline Cervical cancer (Natural health care) & 1.70 & 4.38 & 2.33 & 2.86 & 1.75 & 2.60 \\
\hline Cervical cancer (Bedieman) & 1.40 & 3.75 & 2.50 & 2.29 & 1.75 & 2.34 \\
\hline Endometrial cancer (Bedieman) & 1.40 & 3.75 & 2.50 & 2.29 & 1.75 & 2.34 \\
\hline How to prevent ovarian cancer & 1.70 & 3.88 & 2.33 & 2.57 & 1.13 & 2.32 \\
\hline Ovarian Cancer Awareness & 1.80 & 4.38 & 2.00 & 1.93 & 1.50 & 2.32 \\
\hline Treat prostate cancer & 1.60 & 4.00 & 1.67 & 2.57 & 1.75 & 2.32 \\
\hline Cancer de próstata (Anastore) & 1.60 & 3.50 & 2.17 & 2.71 & 1.50 & 2.32 \\
\hline Cancer de RIÑON & 1.70 & 3.00 & 1.67 & 2.64 & 1.63 & 2.13 \\
\hline Cáncer de vejiga & 1.70 & 3.00 & 1.67 & 2.64 & 1.63 & 2.13 \\
\hline Cancer testicular (Anass apps) & 1.70 & 3.00 & 1.67 & 2.64 & 1.63 & 2.13 \\
\hline Cancer de cuello uterino & 1.70 & 3.00 & 1.67 & 2.64 & 1.63 & 2.13 \\
\hline
\end{tabular}




\begin{tabular}{|c|c|c|c|c|c|c|}
\hline Name of app & Engagement & Functionality & Esthetics & Information & $\begin{array}{l}\text { Subjective } \\
\text { quality }\end{array}$ & Overall \\
\hline Cáncer de ovarios & 1.70 & 3.00 & 2.00 & 2.57 & 1.25 & 2.10 \\
\hline Cáncer uterino & 1.70 & 3.00 & 1.67 & 2.64 & 1.50 & 2.10 \\
\hline El cáncer de vulva & 1.70 & 3.00 & 1.67 & 2.64 & 1.50 & 2.10 \\
\hline Common causes of cervical cancer & 1.80 & 3.13 & 1.50 & 2.50 & 1.50 & 2.09 \\
\hline Ways to treat uterine cancer & 1.60 & 3.50 & 1.33 & 2.00 & 1.63 & 2.01 \\
\hline Cancer cervix fact & 1.40 & 3.63 & 1.33 & 2.14 & 1.25 & 1.95 \\
\hline
\end{tabular}

${ }^{\mathrm{a}}$ For apps with the same name, the developer is indicated in parentheses.

Comparison by the operating system (iOS and Android) revealed an overall MARS score of 3.64 for apps available in the App Store $(\mathrm{n}=15)$ and 2.19 for those available in the Play Store $(\mathrm{n}=40)$; the difference was statistically significant $(P<.001)$. However, when the overall MARS scores were analyzed considering whether the apps were free $(n=41)$ or required payment $(n=5)$, the only significant differences were in the Functionality domain. Comparison by developer type revealed statistically significant differences between the apps that had been supported by a health organization $(n=7)$, with a score of

3.70 , and those that had not $(n=39)$, with a score of 2.85 (Table 4).

Interrater agreement was substantial across all evaluation criteria, except for the Entertainment and Interest criteria of the Engagement domain and the Subjective Quality criterion "What is your overall star rating for the app?" The joint probability of agreement was $>85 \%$ in all the items and $>90 \%$ in 11 of the 23 evaluation criteria and 4 of the 5 domains analyzed (Table 5). The mean $\kappa$ score for the 5 domains was 0.748 , indicating that substantial agreement was observed between the 2 evaluators.

Table 4. Results of the Mobile Application Rating Scale evaluation: comparison by different characteristics.

\begin{tabular}{llllllllll}
\hline Category & \multicolumn{2}{l}{ Operating system } & \multicolumn{3}{l}{ Developer } & & \multicolumn{2}{c}{ Cost } \\
& $\begin{array}{l}\text { Android } \\
(\mathrm{n}=40)\end{array}$ & $\begin{array}{l}\text { iOS } \\
(\mathrm{n}=15)\end{array}$ & $P$ value & $\begin{array}{l}\text { Non-health organiza- } \\
\text { tion }(\mathrm{n}=39)\end{array}$ & $\begin{array}{l}\text { Health organization } \\
(\mathrm{n}=7)\end{array}$ & $\begin{array}{l}P \text { value } \\
\text { Free } \\
(\mathrm{n}=41)\end{array}$ & $\begin{array}{l}\text { Paid } \\
(\mathrm{n}=5)\end{array}$ & $\begin{array}{c}P \text { value } \\
\text { Engagement }\end{array}$ \\
\hline 2.30 & 3.45 & $<.001$ & 2.34 & 3.30 & .01 & 2.40 & 3.24 & .06 \\
Functionality & 3.87 & 4.13 & .32 & 3.85 & 4.63 & .02 & 4.05 & 3.25 & .03 \\
Esthetics & 2.75 & 3.84 & $<.001$ & 2.83 & 3.50 & .10 & 2.86 & 3.53 & .16 \\
Information & 3.00 & 3.72 & $<.001$ & 3.00 & 3.88 & .002 & 3.12 & 3.27 & .67 \\
Subjective quality & 2.18 & 3.05 & .001 & 2.21 & 3.18 & .01 & 2.35 & 2.43 & .88 \\
Overall & 2.82 & 3.64 & $<.001$ & 2.85 & 3.70 & .01 & 2.96 & 3.14 & .62 \\
\hline
\end{tabular}


Table 5. Interrater agreement for the Mobile Application Rating Scale domains and evaluation criteria.

\begin{tabular}{|c|c|c|}
\hline Domains and evaluation criteria & Weighted Cohen $\kappa$ & Agreement (\%) \\
\hline Engagement & 0.76 & 92.2 \\
\hline Entertainment & 0.55 & 89.4 \\
\hline Interest & 0.56 & 87.8 \\
\hline Customization & 0.86 & 96.8 \\
\hline Interactivity & 0.77 & 91.5 \\
\hline Target group & 0.64 & 88.8 \\
\hline Functionality & 0.71 & 90.0 \\
\hline Performance & 0.68 & 89.9 \\
\hline Ease of use & 0.62 & 89.4 \\
\hline Navigation & 0.69 & 87.9 \\
\hline Gestural design & 0.64 & 87.9 \\
\hline Esthetics & 0.80 & 93.6 \\
\hline Layout & 0.75 & 90.8 \\
\hline Graphics & 0.76 & 93.1 \\
\hline Visual appeal & 0.91 & 96.8 \\
\hline Information & 0.79 & 93.6 \\
\hline Accuracy of the app in the description (App/Play Store) & 0.63 & 89.4 \\
\hline Goals & 0.72 & 93.6 \\
\hline Quality of information & 0.67 & 88.7 \\
\hline Quantity of information & 0.65 & 87.2 \\
\hline Visual information & 0.86 & 94.1 \\
\hline Evidence base & 0.66 & 94.1 \\
\hline Credibility & 0.61 & 89.4 \\
\hline Subjective quality & 0.68 & 89.9 \\
\hline Would you recommend this app to people who might benefit from it? & 0.64 & 90.4 \\
\hline Would you pay for this app? & 0.74 & 90.8 \\
\hline $\begin{array}{l}\text { How many times do you think you would use this app in the next } 12 \text { months if it was } \\
\text { relevant to you? }\end{array}$ & 0.72 & 90.4 \\
\hline What is your overall star rating of the app? & 0.48 & 86.7 \\
\hline
\end{tabular}

\section{Discussion}

\section{Principal Findings and Comparison With Previous Work}

Based on a systematic and validated questionnaire (MARS), our study provided an objective ranking of 46 apps for patients with genitourinary cancers available in the Apple and Android stores. Apps for patients with prostate and cervical cancer accounted for almost half of all the apps evaluated (30\% and $17 \%$, respectively). This frequency is consistent with the findings of the Globocan 2018 report [2], according to which genitourinary cancers have the highest global incidence (second in men and fourth in women). Therefore, the number of apps analyzed correlated with the incidence of the specific genitourinary cancers, unlike other types of cancer, such as lung and colorectal cancer, which are extremely prevalent, yet for which few apps have been released [15].
More than half of the apps (61\%) had been updated in the last year and therefore provided better quality information, which is increasingly necessary given the advantages of apps in diagnosis and therapy in this area. Our result is comparable to that obtained in a review of 166 apps for patients with cancer, where it was observed that $52.4 \%$ had been updated in the previous year [15]. However, the proportion of apps that had been developed or promoted by health care organizations was very low $(15.2 \%)$, thus potentially reducing the quality and reliability of the apps. This result was consistent with the conclusions of authors such as Giunti et al [14], who showed an evident absence of health professionals in the development of health care apps. Apps are mostly developed by non-health professionals who are creative and skilled in design but lack scientific knowledge.

Most of the apps included in our study were informative, with generic data on pathophysiology, treatments, and symptoms of 
individual cancers, as reported elsewhere [14]. An important component of some of these apps, such as Treating Bladder Cancer, was including information about the most common symptoms and signs of bladder cancer (hematuria, pain or burning sensation, and increased frequency in urination) that could alert patients to talk to the doctor. These features are important to reduce the risk of having a more advanced stage of cancer. We found that most of the informative apps, like those for cervical cancer, included information about treatment and prognosis. Nonetheless, this information should be given by physicians, and informative apps provide doctors with a tool to improve early diagnosis through successful screening. Additionally, as indicated by Bender et al [23], most of the apps try to increase awareness of cancer in the population. Very few apps focused on how to handle the disease after a diagnosis, correct administration of the treatment prescribed (eg, dosing, management of adverse effects, possible interactions with other long-term medications), and adequate monitoring of symptoms. Only 6 diagnostic apps (13.0\%) were evaluated; of these, 5 were intended for patients with prostate cancer and 1 for patients with testicular cancer. Diagnostic apps are more frequent in other types of cancer, such as melanoma and breast cancer [16].

Several methods for the evaluation of mHealth apps have been developed, although in most cases, the absence of a systematic methodology and the fact that they were not developed by scientific professionals made their routine use impossible [12]. The MARS methodology, as reported by Stoyanov et al [21], is an easy-to-use, simple and logical tool that is considered highly reliable because it is promoted by expert technicians and health care professionals. This evaluation proposes a multidisciplinary analysis designed for all health care apps, with 5 domains covering the main aspects for correct evaluation. Stoyanov et al [13] reported that the MARS questionnaire showed high levels of internal consistency (Cronbach $\alpha=.9$ ) and interrater reliability (two-way mixed intraclass correlation coefficient, 0.79 ; $95 \% \mathrm{CI}, 0.75-0.83$ ) when it was applied to rate 50 mental health apps.

As for the MARS score, our study showed a mean score of 2.98 for the overall quality of the apps, with a score of 3.13 for quality content. These scores were significantly higher than those found by Böhme et al [6] for mobile cancer apps for prostate cancer, breast cancer, and colorectal cancer (1.96). On the other hand, our results were similar to the scores of apps for other diseases. For example, the mean score found by Richardson et al [19] for mobile apps targeted to parents of infants in the neonatal intensive care unit was 3.37, without considering the subjective quality. Salazar et al [24] showed a mean score of 3.92 for mobile apps for the management of pain, while Siddique et al [25] found a median score of 3.70 for apps targeted to the care management of chronic kidney and end-stage renal disease. To note, 22 apps (48\%) scored a value equal to or greater than 3 points (ie, "Acceptable"). Only 5 apps (11\%) exceeded 4 points in the overall quality score (ie, "Good"). Jupp et al [3] found that the apps evaluated stood out in the Functionality domain, with high scores in most of them because they were developed to be highly efficient and easy to use. Consistent with our findings, these authors found that the 3 strategies necessary for optimal use of the smartphone among patients with cancer were the management of symptoms and medications, quality of the information resources, and ability to export data. In contrast, the Esthetics domain had a better correlation with the overall MARS score. It is assumed that this is due to the attempt to develop a more engaging appearance which is directly related to other features, such as a higher frequency of use.

In reports based on MARS for assessment of apps aimed at patients with cancer or other diseases, the domain that scored the lowest was Engagement. The main reason is that the apps were unable to make patients feel that they were participating in the management of their disease. We drew the same conclusion, with Engagement being the domain with the poorest mean score compared to the others. According to the literature, the fundamental aspects that can be improved in this section are customization, user interactivity, and entertainment, which leads to a higher score $[3,20]$. The participation of patients in the development, design, and validation of apps through focus groups considerably improves the score for this domain. Furthermore, the participation of patients in the management of their symptoms by registering and sending messages and reminders significantly enhances the health outcomes [26]. Collado et al [18] found that more than $40 \%$ of patients would be interested in communicating with their physician or pharmacist using an app.

The apps that scored best in the MARS evaluation were "Bladder cancer manager," "Kidney cancer manager," and "My prostate cancer manager." These apps were available in the App Store and stood out because of their high scores in the Engagement and Esthetics domains, as did the next 2 apps in the ranking, "Target Ovarian Cancer Symptoms Diary" and "My Cancer Coach." The "Top 5" apps contained reminders and schedules and offered the possibility of registering analytical information and treatments prescribed, thus enabling a greater score in the Subjective Quality domain because they achieve the main goal. Likewise, the 4 best apps had an explicit preventive purpose, in contrast to the informative purpose that was more frequent in the global analysis.

Of note, 9 of the 10 best apps had been updated during 2018, and 5 of the 10 best apps had been developed by a health care organization. This is an important observation because neither of these 2 aspects is specifically evaluated in MARS. The analysis by the domain of the apps developed by health care organizations and those that were not revealed statistically significant differences for each of the items evaluated. Therefore, the quality of health apps is based on the frequency of updating and the participation of health care organizations in their development, thus confirming the hypothesis proposed elsewhere [15,27]. The first three apps ("Bladder cancer manager," "Kidney cancer manager." and "My prostate cancer manager") had the same developer (Point of Care) which is a platform of health apps intended to patients and clinicians. However, clinicians were not involved in their development. Also, "Target Ovarian Cancer Symptoms Diary" had a clinical advisory panel made up of many oncology specialists who answer patients' questions but they were not involved in promoting the app. Otherwise, "My Cancer Coach" was developed by some health organizations and the coaches formed 
a multidisciplinary team (nurses, physicians...) to provide reliable information to patients. However, the lowest scores were obtained for apps that are merely informative and provide links to other web pages but not their information.

The analysis by operating system revealed a statistically significant difference that was more favorable to the apps from the App Store than those from Play Store, probably because verification requirements for publishing and application are stricter for iOS than for Android.

Apps are beginning to show a significant impact on users' health. Because of that, regulatory authorities are responsible for evaluating these technologies to control their availability in the stores. In 2013 and regarding this issue, the Food and Drug Administration (FDA) published a guide containing recommendations to assess the quality of these apps. However, due to the rise of health apps, an objective, comprehensive and clear evaluation of apps is necessary. This evaluation should allow users and health care professionals (who recommend them to patients) which apps meet minimum standards of quality and safety in their content.

Patients are exposed to unreliable information related to their health so quality certifications are needed to identify those apps that offer the best content for users. For example, App Saludable is a free and open-access certification given to some apps which were developed using strict guidelines in Spain [28]. This is one of the first certifications in Europe that evaluate the quality and safety of health apps.

\section{Limitations}

MARS is limited by its subjectivity $[13,21]$. However, the high interrater reliability obtained between the 2 evaluators, both of whom had experience in the development and validation of health apps and were familiar with genitourinary cancers, highlights the considerable coherence of our results.

\section{Conclusions}

The quality of health apps should be evaluated using approaches such as MARS to decide which apps could be prescribed to patients and to identify which features should be addressed to improve these tools. Most of the apps designed for patients with genitourinary cancers only try to increase awareness and provide data about the disease, without ensuring coherent interactivity. Although the participation of health professionals in the development of these apps is low, we observed that their participation was associated with the app quality and the recency of updates. Greater scores in quality were observed in iOS apps, although no correlation between quality and price was found.

\section{Conflicts of Interest}

None declared.

\section{References}

1. Lopez CM. Avances en supervivencia en tumores genitourinarios gracias a las nuevas técnicas diagnósticas. Gaceta Medica. 2018. URL: https://www.gacetamedica.com/especializada/ avances-en-supervivencia-en-tumores-genitourinarios-gracias-a-las-nuevas-tecnicas-diagnosticas-EG1833350 [accessed 2019-09-19]

2. Bray F, Ferlay J, Soerjomataram I, Siegel RL, Torre LA, Jemal A. Global cancer statistics 2018: GLOBOCAN estimates of incidence and mortality worldwide for 36 cancers in 185 countries. CA Cancer J Clin 2018 Sep 12 [FREE Full text] [doi: 10.3322/caac.21492] [Medline: 30207593]

3. Jupp JCY, Sultani H, Cooper CA, Peterson KA, Truong TH. Evaluation of mobile phone applications to support medication adherence and symptom management in oncology patients. Pediatr Blood Cancer 2018 Nov;65(11):e27278. [doi: 10.1002/pbc.27278] [Medline: 29943893]

4. Ali EE, Chan SSL, Leow JL, Chew L, Yap KY. User acceptance of an app-based adherence intervention: Perspectives from patients taking oral anticancer medications. J Oncol Pharm Pract 2019 Mar 23;25(2):390-397 [FREE Full text] [doi: 10.1177/1078155218778106] [Medline: 29792123]

5. Jiang Y, West BT, Barton DL, Harris MR. Acceptance and Use of eHealth/mHealth Applications for Self-Management Among Cancer Survivors. Stud Health Technol Inform 2017;245:131-135 [FREE Full text] [Medline: 29295067]

6. Böhme C, von Osthoff MB, Frey K, Hübner J. Qualitative evaluation of mobile cancer apps with particular attention to the target group, content, and advertising. J Cancer Res Clin Oncol 2018 Jan 19;144(1):173-181. [doi:

10.1007/s00432-017-2533-0] [Medline: 29052039]

7. Owens OL, Beer JM, Reyes LI, Thomas TL. Systematic Review of Commercially Available Mobile Phone Applications for Prostate Cancer Education. Am J Mens Health 2019 Dec 07;13(1):1557988318816912 [FREE Full text] [doi: 10.1177/1557988318816912] [Medline: 30526243]

8. Adam A, Hellig JC, Perera M, Bolton D, Lawrentschuk N. 'Prostate Cancer Risk Calculator' mobile applications (Apps): a systematic review and scoring using the validated user version of the Mobile Application Rating Scale (uMARS). World J Urol 2018 Apr 8;36(4):565-573. [doi: 10.1007/s00345-017-2150-1] [Medline: 29222595]

9. Sawesi S, Rashrash M, Phalakornkule K, Carpenter JS, Jones JF. The Impact of Information Technology on Patient Engagement and Health Behavior Change: A Systematic Review of the Literature. JMIR Med Inform 2016 Jan 21;4(1):e1 [FREE Full text] [doi: 10.2196/medinform.4514] [Medline: 26795082]

10. The Growing Value of Digital Health: Evidence and Impact on Human Health and the Healthcare System Institute Report. IQVIA. 2017. URL: https://www.iqvia.com/institute/reports/the-growing-value-of-digital-health [accessed 2019-09-03] 
11. Boyle JA, Xu R, Gilbert E, Kuczynska-Burggraf M, Tan B, Teede H, et al. Ask PCOS: Identifying Need to Inform Evidence-Based App Development for Polycystic Ovary Syndrome. Semin Reprod Med 2018 Dec;36(1):59-65. [doi: 10.1055/s-0038-1667187] [Medline: 30189452]

12. Stec MA, Arbour MW, Hines HF. Client-Centered Mobile Health Care Applications: Using the Mobile Application Rating Scale Instrument for Evidence-Based Evaluation. J Midwifery Womens Health 2019 May 18;64(3):324-329. [doi: 10.1111/jmwh.12941] [Medline: 30887711]

13. Stoyanov SR, Hides L, Kavanagh DJ, Zelenko O, Tjondronegoro D, Mani M. Mobile app rating scale: a new tool for assessing the quality of health mobile apps. JMIR mHealth uHealth 2015 Mar;3(1):e27 [FREE Full text] [doi: 10.2196/mhealth.3422] [Medline: 25760773]

14. Giunti G, Giunta DH, Guisado-Fernandez E, Bender JL, Fernandez-Luque L. A biopsy of Breast Cancer mobile applications: state of the practice review. Int J Med Inform 2018 Dec;110:1-9 [FREE Full text] [doi: 10.1016/j.ijmedinf.2017.10.022] [Medline: 29331247]

15. Jongerius C, Russo S, Mazzocco K, Pravettoni G. Research-Tested Mobile Apps for Breast Cancer Care: Systematic Review. JMIR mHealth uHealth 2019 Feb 11;7(2):e10930 [FREE Full text] [doi: 10.2196/10930] [Medline: 30741644]

16. Collado-Borrell R, Escudero-Vilaplana V, Ribed-Sánchez A, Ibáñez-García S, Herranz-Alonso A, Sanjurjo-Sáez M. Smartphone applications for cancer patients; what we know about them? Farm Hosp 2016 Jan 01;40(1):25-35 [FREE Full text] [doi: 10.7399/fh.2016.40.1.8993] [Medline: 26882831]

17. Crico C, Renzi C, Graf N, Buyx A, Kondylakis H, Koumakis L, et al. mHealth and telemedicine apps: in search of a common regulation. Ecancermedicalscience 2018;12:853 [FREE Full text] [doi: 10.3332/ecancer.2018.853] [Medline: 30079115]

18. Berkowitz CM, Zullig LL, Koontz BF, Smith SK. Prescribing an App? Oncology Providers' Views on Mobile Health Apps for Cancer Care. JCO Clinical Cancer Informatics 2017 Nov(1):1-7. [doi: 10.1200/CCI.17.00107] [Medline: 29113731]

19. Richardson B, Dol J, Rutledge K, Monaghan J, Orovec A, Howie K, et al. Evaluation of Mobile Apps Targeted to Parents of Infants in the Neonatal Intensive Care Unit: Systematic App Review. JMIR mHealth uHealth 2019 Apr 15;7(4):e11620 [FREE Full text] [doi: 10.2196/11620] [Medline: 30985282]

20. Kessel KA, Vogel MM, Kessel C, Bier H, Biedermann T, Friess H, et al. Mobile Health in Oncology: A Patient Survey About App-Assisted Cancer Care. JMIR mHealth uHealth 2017 Jun 14;5(6):e81 [FREE Full text] [doi: 10.2196/mhealth.7689] [Medline: 28615159]

21. Stoyanov SR, Hides L, Kavanagh DJ, Wilson H. Development and Validation of the User Version of the Mobile Application Rating Scale (uMARS). JMIR mHealth uHealth 2016 Jun 10;4(2):e72 [FREE Full text] [doi: 10.2196/mhealth.5849] [Medline: 27287964]

22. Moher D, Shamseer L, Clarke M, Ghersi D, Liberati A, Petticrew M, et al. Preferred reporting items for systematic review and meta-analysis protocols (PRISMA-P) 2015 statement. Syst Rev 2015 Jan 01;4:1 [FREE Full text] [doi: 10.1186/2046-4053-4-1] [Medline: 25554246]

23. Bender JL, Yue RYK, To MJ, Deacken L, Jadad AR. A lot of action, but not in the right direction: systematic review and content analysis of smartphone applications for the prevention, detection, and management of cancer. J Med Internet Res 2013 Dec;15(12):e287 [FREE Full text] [doi: 10.2196/jmir.2661] [Medline: 24366061]

24. Salazar A, de Sola H, Failde I, Moral-Munoz JA. Measuring the Quality of Mobile Apps for the Management of Pain: Systematic Search and Evaluation Using the Mobile App Rating Scale. JMIR mHealth uHealth 2018 Oct 25;6(10):e10718 [FREE Full text] [doi: $\underline{10.2196 / 10718]}$ [Medline: $\underline{\text { 30361196] }}$

25. Siddique AB, Krebs M, Alvarez S, Greenspan I, Patel A, Kinsolving J, et al. Mobile Apps for the Care Management of Chronic Kidney and End-Stage Renal Diseases: Systematic Search in App Stores and Evaluation. JMIR mHealth uHealth 2019 Sep 04;7(9):e12604 [FREE Full text] [doi: 10.2196/12604] [Medline: 31486408]

26. Pérez-Jover V, Sala-González M, Guilabert M, Mira JJ. Mobile Apps for Increasing Treatment Adherence: Systematic Review. J Med Internet Res 2019 Jun 18;21(6):e12505 [FREE Full text] [doi: 10.2196/12505] [Medline: 31215517]

27. Cantudo-Cuenca M, Robustillo-Cortés M, Cantudo-Cuenca M, Morillo-Verdugo R. A better regulation is required in viral hepatitis smartphone applications. Farm Hosp 2014 Apr 01;38(2):112-117 [FREE Full text] [Medline: 24669895]

28. Apps i projectes de mobilitat. TicSalut. URL: https://ticsalutsocial.cat/es/ [accessed 2020-02-01]

\section{Abbreviations}

FDA: Food and Drug Administration

MARS: Mobile Application Rating Scale 
Edited by G Eysenbach; submitted 27.12.19; peer-reviewed by T Muto, M Gaman; comments to author 27.01.20; revised version received 06.02.20; accepted 23.03.20; published 23.07.20

Please cite as:

Amor-García MÁ, Collado-Borrell R, Escudero-Vilaplana V, Melgarejo-Ortuño A, Herranz-Alonso A, Arranz Arija JÁ, Sanjurjo-Sáez $M$

Assessing Apps for Patients with Genitourinary Tumors Using the Mobile Application Rating Scale (MARS): Systematic Search in App Stores and Content Analysis

JMIR Mhealth Uhealth 2020;8(7):e17609

URL: http://mhealth.jmir.org/2020/7/e17609/

doi: 10.2196/17609

PMID: 32706737

CMiguel Ángel Amor-García, Roberto Collado-Borrell, Vicente Escudero-Vilaplana, Alejandra Melgarejo-Ortuño, Ana Herranz-Alonso, José Ángel Arranz Arija, María Sanjurjo-Sáez. Originally published in JMIR mHealth and uHealth (http://mhealth.jmir.org), 23.07.2020. This is an open-access article distributed under the terms of the Creative Commons Attribution License (https://creativecommons.org/licenses/by/4.0/), which permits unrestricted use, distribution, and reproduction in any medium, provided the original work, first published in JMIR mHealth and uHealth, is properly cited. The complete bibliographic information, a link to the original publication on http://mhealth.jmir.org/, as well as this copyright and license information must be included. 